\title{
VIBRATING WIRE SCANNER FOR BEAM PROFILE MONITORING
}

\author{
Arutunian S.G., Dobrovolski N.M., Mailian M.R., Sinenko I.G., Vasiniuk I.E. \\ Yerevan Physics Institute, Br. Alikhanian St. 2, 375062 Yerevan, Armenia
}

Abstract

Method of beam transverse profile measurement in accelerators by scanning wire is wide-spread in accelerator technology [1-6]. In this work vibrating wire is proposed to use as a scanning wire. In such way the system of radiation (or secondary particles) extraction and measurement can be removed. Dependence of oscillations frequency on beam scattering is determined by several factors. Our estimations show that influence caused by wire heating will dominate.

\section{INTRODUCTION}

A beam of charged particles affects on vibrating wire frequency in several ways: mechanical transverse influence caused by transferred pulse of the beam, influence of beam magnetic field, radiation affect of the beam on the wire material, heating of the wire. The last effect is dominating, since for typical currents of accelerator beams the wire of $\mu \mathrm{m}$ diameter heats over $1000 \mathrm{~K}[2,3]$.

Authors have accumulated a certain experience in development of tension gauges on the basis of vibrating wire. Frequency long-time relative stability $\sim 10^{-5}$ at relative resolution $\sim 10^{-6}$ is obtained. As a result of frequency multiplication its measurement become much more accurate. We have used such pickups for measurement of spatial distribution of magnetic field [7].

\section{EQUILIBRIUM TEMPERATURE}

Consider a long thin round wire, strained along the $y$ axis. Wire ends are kept at constant temperature $T_{0}$. "Thin" and "long" mean that the wire diameter $d$, its length $l$ and part of the wire irradiated by the beam $\sigma_{y}$ satisfy the condition $d<<\sigma_{y}<l$. Beam propagates along the $z$ axis, the wire scans the beam in transverse $x$ direction.

Equilibrium temperature of an immobile wire in vacuum under beam is defined by the balance between the power $\Delta E / \Delta t$ brought to it by the beam and two factors of heat removal: thermal radiation through the surface $W_{1}$ and thermoconductivity of the wire material through its ends $W_{2}$.

The power released on the wire can be estimated by formula:

$$
\frac{\Delta E}{\Delta t}=k\left(\frac{d E}{d z} d\right)\left(p(x) \sigma_{y} d\right),
$$

where $d E / d z$ is the ionisation loss of beam particles in wire material, $p(x)$ is local flux density of beam particles, $k$ is the coefficient characterising the part of scattered beam energy working on wire heating. In works $[3,4] k$ is set equal to $1 / 3$.

The thermal radiation $W_{1}$ is determined by the formula:

$$
W_{l}=S_{\text {side }} \sigma\left(T^{4}-T_{0}^{4}\right)
$$

where $S_{\text {side }}$ is the side surface of the wire, $\sigma$ is the constant of Stefan-Boltzmann, $T$ is the absolute temperature of the wire.

Heat loss through the wire ends approximately is equal

$$
W_{2}=2 S K\left(T-T_{0}\right) / l,
$$

where $S$ is the wire cross section, $K$ is the thermal conductivity of wire material.

Fig. 1 shows calculated curves $W_{1}, W_{2}$ and $W_{1}+W_{2}\left(\sigma_{y}=1 \mathrm{~mm}\right)$.

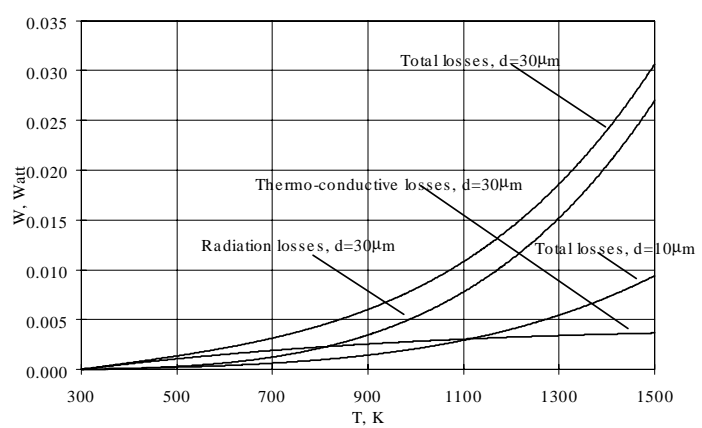

Fig. 1. Energy losses for tungsten wire, $\mathrm{d}_{1}=30 \mu \mathrm{m}$, $\mathrm{d}_{2}=10 \mu \mathrm{m}, \mathrm{l}=40 \mathrm{~mm}, \sigma_{\mathrm{x}}=3 \mathrm{~mm}, \sigma_{\mathrm{z}}=1 \mathrm{~mm}$. 
The equilibrium temperature of the wire is determined from the equation of balance:

$$
W_{1}+W_{2}=\Delta E / \Delta t \text {. }
$$

Solution of this equation with respect to $T$ at different distances of the wire from the beam centre are presented in Fig. 2.

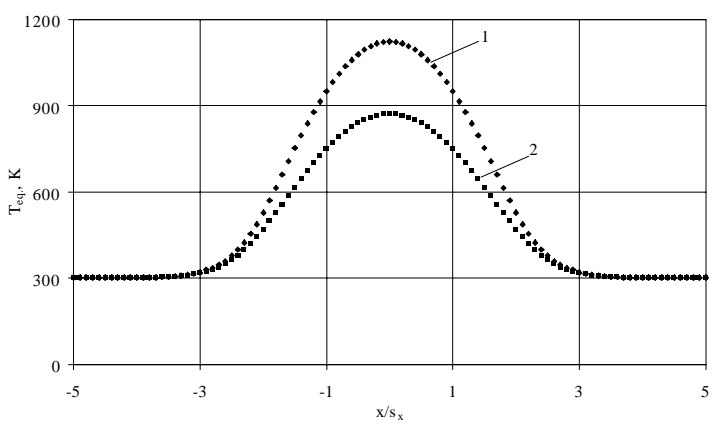

Fig. 2. Wire equilibrium temperature, $d=30 \mu \mathrm{m}$ (1), $\mathrm{d}=10 \mu \mathrm{m}(2)$.

Time of thermalisation $\tau \cong E_{\text {store }} / W$ also is an important parameter for the scanning, where $E_{\text {store }}$ is the thermal energy stored in the wire. Calculations show that the thermalisation time is minimal in the beam centre and is 2 and 3 seconds respectively for tungsten wires of diameters $10 \mu \mathrm{m}$ and $30 \mu \mathrm{m}$. In the beam periphery this time is approximately doubled.

Note when scanning consecutively the total time of scanning is much more less than the sum of thermalisation times of individual points, because of the wire temperature changes insignificantly at passing from one point to another.

\section{MODEL EXPERIMENT}

Influence of the wire temperature on its natural oscillations frequency is determined by its elongation:

$$
\Delta f / f=-(1 / 2) \Delta l / l=\alpha_{b} \Delta T,
$$

where $\Delta T$ is the temperature variation. Coefficient $\alpha_{b}$ for metals varies from few units of $10^{-6} \mathrm{~K}$ up to $10^{-5} \mathrm{~K}^{-1}$ (steels, bronzes, brasses, aluminium alloys).

However, for the rigidly fixed wire more essential is the influence of temperature changes on wire tension arising from inequality of coefficients of thermal expansion of wire $\alpha_{s}$ and the base $\alpha_{b}$ :

$$
\frac{\Delta f}{f}=\frac{1}{2} \frac{\Delta F}{F} \approx \frac{E S}{F}\left(\alpha_{b}-\alpha_{s}\right) \Delta T,
$$

where $E$ is the Young's modulus, $F$ is the wire tension. In formula (7) a large dimensionless factor $E S / F$ is separated out. Thus, for the wire with rigidly fixed ends the dominating factor is the influence of the temperature on wire tension.

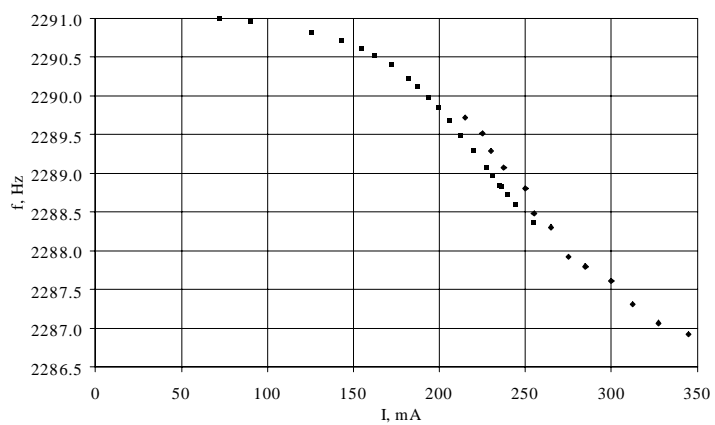

Fig. 3. Frequency dependence on current.

Heating of the scanning wire was modelled by passing of direct currents through the wire. Wire natural oscillations were generated in two modes: with one free end and with fixed ends of the wire.

Results of two series of measurements for tungsten wire of diameter $70 \mu \mathrm{m}$ are presented in Fig. 3. The first series (squares) was carried out for currents from $75 \mathrm{~mA}$ up to $250 \mathrm{~mA}$ (glow appeared at $235 \mathrm{~mA}$ ) and from $215 \mathrm{~mA}$ up to $345 \mathrm{~mA}$. In first series the current was changed stepwise. In second case between two consequent values the current was nullified. In second series the frequency had restored well at each switching on for currents up to $345 \mathrm{~mA}$. Yellow glow ( $T \approx 1000 \mathrm{~K}$ ) appears for currents more than $300 \mathrm{~mA}$.

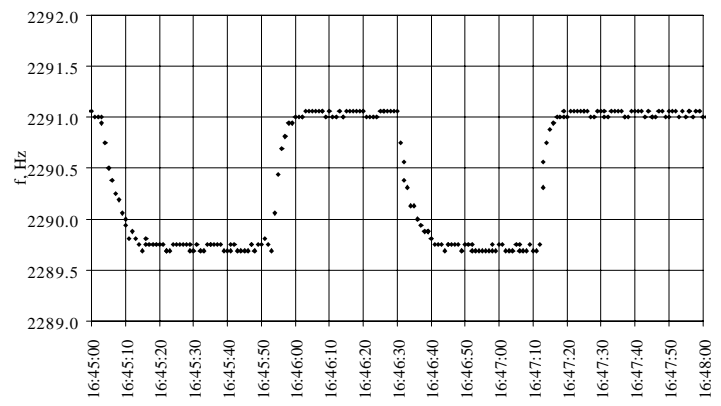

Fig. 4. Transient characteristics of wire with a free end, $I=215 \mathrm{~mA}$.

Fig. 4 shows the transient characteristics at switching on/off for the current $215 \mathrm{~mA}$. One can see that the frequency exceeds its steady-state value during a few seconds. One can take this value for estimate of the thermalisation time $\tau$. Cooling down processes are depicted without destruction, because after switching on it takes some time for current to reach its steady-state. 


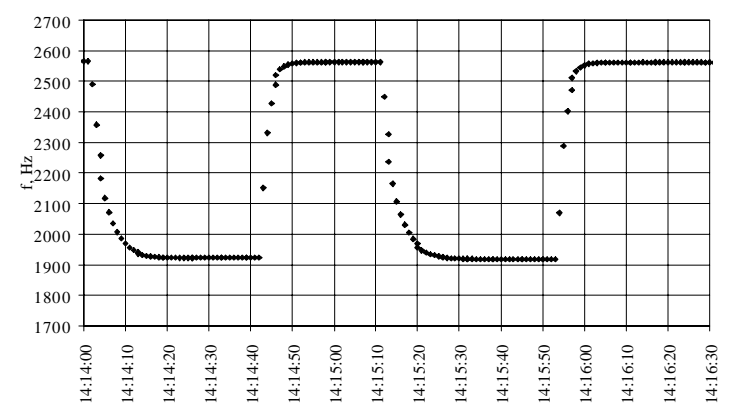

Fig. 5. Transient characteristics of wire with rigidly fixed ends, $I=215 \mathrm{~mA}$.

Similar experiments were done for wires with rigidly fixed ends. As it was mentioned above, the frequency changes interval was much more wider. Fig. 5 shows the transient characteristics at switching on/off of the current $215 \mathrm{~mA}$.

Restoration of the frequency in both cases were good enough, i.e. heating of the wire up to $1000 \mathrm{~K}$ does not lead to irreversible changes of wire parameters.

\section{MAGNETIC FIELD}

There are two possibilities in scheme of vibrating wire scanner: to use a scheme with own magnet or use the accelerator magnetic fields (dipole magnets, quadrupole lenses). In developed by the authors pickups natural oscillations of the wire were excited in autogeneration scheme. Exciting action on the wire arises as a result of interaction between the current through the wire and magnetic field of the samarium cobalt magnet, the field being localised in an magnet operating gap of the length $10 \mathrm{~mm}$. The characteristic field induction inside the gap was about $8 \mathrm{kGs}$.

To keep the transverse effective square of the wire with respect to on-going bunch, separation of oscillations along the bunch axis is desirable.

Fields of dipole magnets are strong enough, however, they fix the horizontal movement of the wire. It is also possible to use the fields of quadrupole lenses. Scanning can be done in vertical, horizontal or under the angle $45^{\circ}$ directions.

Usage of vibrating wire scanners with own magnets located in accelerators free spaces is also possible. In so doing it is preferable to intersect the bunch at $1 / 4$ of wire length, the magnetic circuit being located symmetrically with respect to wire centre, in such way providing generation of the second harmonic. In this case the magnetic field outside the gap is to be carefully screened.

\section{THERMALISATION TIMES}

Stability with respect to wire heating is one of the main problems in developed wire scanner technologies. To solve this problem, the scanning was done at a high speed, up to $\approx 10 \mathrm{~m} / \mathrm{sec}$. [3, 4], i.e. the wire passes across the $\mathrm{mm}$ size bunch during $10^{-4}$ seconds. Such times are too small for thermalisation of the wire. Note, the estimate of $\tau$ can be lowered, because in real movement from one point to another will result in less temperature jumps. However, one cannot expect thermalisation times less than $10^{-2} \mathrm{~s}$ at temperatures, where long time exploitation of wire is possible.

It is possible that problem of thermalisation time can be solved by improvement of wire fixing system, which now is essentially simplified in comparison with similar wire scanning systems.

\section{CONCLUSION}

Indubitable advantage of proposed method is the compactness of the whole system and the elimination of the unit of radiation receivers. The peripheral monitoring of the bunch by proposed method also can be interesting.

Authors would like to thank R.Reetz and A.Ts.Amatuni for helping support.

\section{REFERENCES}

1. Suwada T. et al. First beam test result of a prototype wire scanner for the KEKB injector linac and BT lines. - KEK Preprint 97-184, 1997.

2. Steinbach $\mathrm{Ch}$. Emmitance measurements with the CERN PS wire scanner. - CERN/PS 95-04(OP).

3. Agoritsas V. et al. The first wire scanner of the CERN PS, CERN/PS 95-06 (BD/OP).

4. Fulton R. et al. A high resolution wire scanner for micron-size profile measurements of the SLC. - SLAC-PUB-4605, 1988.

5. Field C. The wire scanner system of the Final Focus Test Beam. - SLAC-PUB-6717, 1994.

6. McCormick D. et al. Measuring micron size beams in the SLC Final Focus, - SLAC-PUB-6615, 1994.

7. Arutunian S.G. et al, Magnetic field distribution measurement by vibrating wire strain gauge.- PAC'99. 